\title{
Realizations of the Solution of the Total Schrödinger Equation from the Link Between Relativity and Quantum Mechanics
}

\author{
Mohamed Daris* \\ Department of Physics, University of Sciences, Morocco
}

\author{
*Corresponding Author: Mohamed Daris, Department of Physics, University of Sciences, Morocco
}

\begin{abstract}
This work is due for the solution of the big problem which is the solution of the Schrödinger equation to have a new model or system that can have other consequences on the whole matter which leads to a resolution of all the quantum problems to link to physics, which makes theories in quantum physics easier to answer on major and complex questions. The solution of the Schrödinger equation is based on the connection between quantum mechanics and relativity. It is a drift of this connection so we have this system is completely solved and gives a great interpretation of all the particles and waves and all macroscopic or microscopic systems eg galaxies; the planets ; the moons etc. for example, particles, waves, electrons, protons, etc. will be noted.
\end{abstract}

Keywords: Schrödinger equation; variable; wave function; energy.

\section{ABREVIATIONS :}

$\rho$ : density; E: energy of light, $\psi$ : wave function, $\mathrm{T}_{\mathrm{F}}$ : final time, $\mathrm{H}$ : own operator, $\mathrm{E}_{\mathrm{V}}$ : volume energy, C: speed of light, D $(\psi)$ : function of the function variable of wave.

\section{INTRODUCTION}

The solution of the Schrödinger equation that I exploit is based on the connection between quantum mechanics and relativity that I already exploit in another subject that gives us a new equation that is a function of the function of It is at this level that we have tried to derive this solution because this link is the basis of all practical solutions and systems. I have tried to calculate the function of the solution which is continuous normal and according to the wave function it allows a global study and localization of the solution to allow to pass to other more important and more complex level.

\section{DISCUSSION}

\section{I) Relationship building for the model $\left(\mathbf{E}_{m}, \mathbf{E}_{\rho}, \mathbf{E}_{\mathrm{V}}\right)$ :}

We have:

$$
E=\Psi C^{2} \sqrt{T_{F}} \quad ; \quad m=\frac{E}{C^{2} \sqrt{T_{F}}}=\frac{\Psi C^{2} \sqrt{T_{F}}}{\sqrt{\rho}} \times \frac{1}{C^{2} \sqrt{T_{F}}}=\frac{\Psi}{\sqrt{\rho}}
$$

And we know that: $m=\frac{\Psi}{\sqrt{\rho}}$

So: $\rho=\frac{\Psi^{2}}{m^{2}} \quad ; \quad V=\frac{m}{\rho}=\frac{\Psi}{\sqrt{\rho}}=\frac{m^{2}}{\Psi^{2}}=\frac{m^{2}}{\sqrt{\rho} \Psi}$

$m=\frac{\Psi}{\sqrt{\rho}} \quad$;

$\rho=\frac{\Psi^{2}}{m^{2}} \quad ;$

$V=\frac{m^{2}}{\sqrt{\rho} \Psi} \quad ;$

- Model design $\left(\mathrm{E}_{\mathrm{m}}, \mathrm{E}_{\rho}, \mathrm{E}_{\mathrm{V}}\right)$ :

We have: 


$$
m=\frac{E}{C^{2} \sqrt{T_{F}}}=\frac{\Psi}{\sqrt{\rho}} \quad ; \quad \rho=\left(\frac{\Psi \mathrm{c}^{2}}{E}\right)^{2} \times T_{F}=\frac{\Psi^{2}}{m^{2}} \quad ; \quad V=\frac{E}{\mathrm{c}^{2} \Psi^{2} \sqrt{T_{F}}}=\frac{m^{2}}{\sqrt{\rho} \Psi}
$$

For $\mathrm{E}_{\mathrm{m}}$ we have:

$\frac{E}{C^{2} \sqrt{T_{F}}}=\frac{\Psi}{\sqrt{\rho}}$

So:

$$
\begin{gathered}
E=\frac{\Psi \mathrm{c}^{2} \sqrt{T_{F}}}{\sqrt{\rho}} \\
E_{m}=\Psi \mathrm{c}^{2}\left(\frac{T_{F}}{\rho}\right)^{\frac{1}{2}}
\end{gathered}
$$

For $\mathrm{E}_{\rho}$ we have:

$$
\left(\frac{\Psi \mathrm{c}^{2}}{E}\right)^{2} \times T_{F}=\frac{\Psi^{2}}{m^{2}}
$$

So:

$$
\frac{\Psi C^{4}}{E^{2}} \times T_{F}=\frac{\Psi^{2}}{m^{2}}
$$

$E_{\rho}=m c^{2} \sqrt{T_{F}}$

So: $E_{\rho}=\frac{\Psi}{\sqrt{\rho}} c^{2} \sqrt{T_{F}}=\Psi \mathrm{c}^{2}\left(\frac{T_{F}}{\rho}\right)^{\frac{1}{2}}$

$E_{\rho}=\Psi \mathrm{c}^{2}\left(\frac{T_{F}}{\rho}\right)^{\frac{1}{2}}$

For $\mathrm{E}_{\mathrm{v}}$ we have:

$$
V=\frac{E}{c^{2} \Psi^{2} \sqrt{T_{F}}}=\frac{m^{2}}{\sqrt{\rho} \Psi}
$$

So: $E=m^{2} c^{2} \Psi\left(\frac{T_{F}}{\rho}\right)^{\frac{1}{2}}$

So: $E_{V}=m^{2} c^{2} \Psi\left(\frac{T_{F}}{\rho}\right)^{\frac{1}{2}}$

So the 3 final results are:

$$
\begin{gathered}
E_{m}=\Psi \mathrm{c}^{2}\left(\frac{T_{F}}{\rho}\right)^{\frac{1}{2}} ; \\
E_{\rho}=\Psi \mathrm{c}^{2}\left(\frac{T_{F}}{\rho}\right)^{\frac{1}{2}} ; \\
E_{V}=m^{2} \mathrm{c}^{2} \Psi\left(\frac{T_{F}}{\rho}\right)^{\frac{1}{2}} ;
\end{gathered}
$$

We can deduce that: $E_{m}=E_{\rho}=\Psi \mathrm{c}^{2}\left(\frac{T_{F}}{\rho}\right)^{\frac{1}{2}}$

So we have: $E_{V}=E_{\rho} m^{2}=E_{m} m^{2}$

- these results show that volume-related energy $E_{V}$ is very large compared to the energy related to the mass energy $E_{m}$ or density $E_{\rho}$ which are identical.

We have: $\rho=\frac{\Psi^{2}}{\mathrm{~m}^{2}}$ 
so: $m^{2}=\frac{\Psi^{2}}{\rho}$

So we will have the new relationship:

$E_{V}=m^{2} c^{2} \Psi\left(\frac{T_{F}}{\rho}\right)^{\frac{1}{2}}=\frac{\Psi^{3} c^{2}}{\rho}\left(\frac{T_{F}}{\rho}\right)^{\frac{1}{2}}$

So: $E_{V}=\frac{\Psi^{3} \mathrm{c}^{2} \sqrt{T_{F}}}{(\rho)^{\frac{3}{2}}}$

- We have: $E_{V}=E_{\rho} m^{2}=E_{m} m^{2}$

so: $E_{V}=E_{\rho} \frac{\Psi^{2}}{\rho}=E_{m} \frac{\Psi^{2}}{\rho}$

So we have the stability of the total matter:

$\rho E_{V}=E_{\rho} \Psi^{2}$

And we have:

$\rho E_{V}=E_{m} \Psi^{2}$

So we have the density of matter is exist in two forms of energy: mass energy and energy density. In a global form of energy, which is the energy density, we have: $\rho=\frac{E_{\rho} \Psi^{2}}{E_{V}}=\frac{E_{m} \Psi^{2}}{E_{V}}$

- This relation is the total stable energy equilibrium relation of matter or of a body; system; universe ... that has an energy $\mathrm{E}_{\mathrm{m}} ; \mathrm{E}_{\rho} ; \mathrm{E}_{\mathrm{V}}$. This relationship represents a link between quantum mechanics and relativity.

The final equation of the connection between relativity and quantum mechanics is:

We pose:

$E_{\rho}=E_{m}=E$

So we will have:

$\rho=\frac{E \Psi^{2}}{E_{V}}$

So the final equation is this: $E=\frac{\rho E_{V}}{\Psi^{2}}$

From the equation linking relativity and quantum mechanics we have: $E=\frac{\rho E_{V}}{\Psi^{2}}$

We have the Schrödinger equation: $H \Psi=E \Psi$

So we have:

$E=\frac{\rho E_{V}}{\Psi^{2}}$

So :

$E \Psi=\frac{\rho E_{v}}{\Psi}$

We know that :

$E \Psi=H \Psi$

so :

$E \Psi=H \Psi=\frac{\rho E_{v}}{\Psi}$ 
We know that $: \rho=\frac{\Psi^{2}}{m^{2}}$

so :

$E \Psi=H \Psi=\frac{\Psi E_{v}}{\mathrm{~m}^{2}}$

So we will have a consequence of:

$E \Psi=H \Psi=\frac{\Psi E_{v}}{\mathrm{~m}^{2}}$

So the exact solution is:

$E \Psi=H \Psi=\frac{E_{v}}{m^{2}} \Psi$

Other result:

We know that :

$E_{v}=m^{2} c^{2} \Psi\left(\frac{T_{F}}{\rho}\right)^{1 / 2}$

so :

$E \Psi=H \Psi=\frac{m^{2} c^{2} \Psi^{2}}{m^{2}}\left(\frac{T_{F}}{\rho}\right)^{1 / 2}$

So we have:

$E \Psi=H \Psi=\left[c^{2}\left(\frac{T_{F}}{\rho}\right)^{1 / 2}\right] \Psi^{2}$

We put the function:

$D(\Psi)$ : Function of the wave function variable: $(\Psi)$ :

We have : $E \Psi=H \Psi=\frac{\rho E_{V}}{\Psi}=\frac{\Psi E_{V}}{m^{2}}$

so : $E \Psi=H \Psi=D(\Psi)$

With :

$D(\Psi)$ Function of the wave function variable: $(\Psi) . D(\Psi)=\frac{\rho E_{V}}{\Psi}=\frac{\Psi E_{V}}{m^{2}}$

$D(\Psi)$ : This is the exact solution of the Schrödinger equation. It is a function of the wave function variable ( $\Psi)$ that allows us to simply exploit another horizon in wave and particle physics and physics quantum. This solution is a special solution of the Schrödinger equation in the state and quantum conditions of particles and quanta.

\section{REFERENCES}

[1] Ferrarese, Laura; Merritt, David (2000). "A fundamental relation between supermassive black holes and their host galaxies". Astrophysical Journal Letters. 539 (1):L9L12. arXiv:astroph/0006053 . Bibcode: 2000ApJ...539L...9F. doi:10.1086/312838.

[2] S. Lloyd, «Ultimate physical limits of computation », Nature, vol. 406, no 6799, 2000, p. 10471054 (PMID 10984064,DOI 10.1038/35023282, Bibcode 2000Natur.406.1047L, arXiv quant-ph/9908043)

[3] Laura Merritt et David Merritt, «A fundamental relation between supermassive black holes and their host galaxies », Astrophysical Journal L12 (DOI 10.1086/312838, Bibcode 2000ApJ...539L...9F,arXiv astro-ph/0006053)

[4] "Speed of light in vacuum, c, c0". The NIST reference on constants, units, and uncertainty: Fundamental physical constants. NIST. Retrieved 2011-11-28. 
[5] Ishimaru, H (1989). "Ultimate Pressure of the Order of 10-13 torr in an Aluminum Alloy Vacuum Chamber". Journal of Vacuum Science and Technology. 7 (3-II): 2439-2442. doi:10.1116/1.575916.

[6] Altarelli, Guido (2008). "Chapter 2: Gauge theories and the Standard Model". Elementary Particles: Volume 21/A of Landolt-Börnstein series. Springer. pp. 2-3. ISBN 3-540-74202-6. The fundamental state of minimum energy, the vacuum, is not unique and there are a continuum of degenerate states that altogether respect the symmetry...

Citation: Mohamed Daris, Realizations of the Solution of the Total Schrödinger Equation from the Link Between Relativity and Quantum Mechanics, International Journal of Scientific and Innovative Mathematical Research (IJSIMR), vol. 8, no. 6, pp. 6-10, 2020. Available : DOI: https://doi.org/10.20431/23473142.0806002

Copyright: (C) 2020 Authors. This is an open-access article distributed under the terms of the Creative Commons Attribution License, which permits unrestricted use, distribution, and reproduction in any medium, provided the original author and source are credited. 